\title{
Natura@economía
}

ISSN 2226-9479 (Versión electrónica)

\section{Potencialidades de la puesta en valor del área natural protegida de Choquequirao en Cusco} Potential of valuating the natural conservation protected area of Choquequirao in
Cusco

\author{
Crispin Cunya Marianella ${ }^{1}$; Luis Alberto Jiménez Diaz ${ }^{1 *}$
}

${ }^{1}$ Universidad Nacional Agraria La Molina, Lima, Perú. Email: jdl@lamolina.edu.pe

Recepción: 5/05/2018; Aceptación: 15/12/2018

\begin{abstract}
Resumen
El complejo arqueológico de Choquequirao, es un asentamiento de la época inca, y es conocido como la hermana sagrada de Machu Picchu por la semejanza estructural y arquitectónica, y recién en el presente milenio, se comenzaron las intervenciones para su recuperación y puesta en valor del monumento, lo que se ha reflejado en el aumento del flujo turístico hacia Choquequirao. A pesar de las mejoras de acceso y de su infraestructura, aún el complejo no cuenta con un nivel de visitas relevante y entre los retos de los próximos años está en realizar acciones para la puesta en valor de Choquequirao que permita que el desarrollo económico de la región sea compatible con la conservación del patrimonio cultural y natural, siendo la planificación y políticas fundamentales para lograr este propósito.
\end{abstract}

Palabras clave: Desarrollo sostenible; Áreas Naturales Protegidas; Políticas ambientales; Cusco.

\begin{abstract}
The archaeological Complex Choquequirao exists since the Inca Civilization and is known as the sacred sister of Machu Picchu because his structural and arquitechtonic similarity, but only since the last millennium began the investments for improving the access to the complex that has lead to an increase of the touristic flows to the zone. Nevertheless, the complex has a reduced number of visitors and the challenge is to increase the potential value of Choquequirao, sharing the economic with the environmental and cultural development of the complex, and the public policies could are quite important for reaching those objectives.
\end{abstract}

Keywords: Sustainable Development; Natural Protected Areas; Environmental policies; Cusco.

Forma de citar el artículo: Cunya, C.; Jiménez, L. 2018. Potencialidades de la puesta en valor del área natural protegida de Choquequirao en Cusco. Natura@economía 3(2):45-60(2018). 


\section{Introducción}

Las Áreas Naturales Protegidas (ANP) tienen por característica su belleza paisajística, su alta concentración de biodiversidad y son patrimonio cultural. Constituyen espacios atractivos para el turismo y oportunidades de generar ingresos para la conservación; sin embargo, los sectores respectivos del Estado aún no vienen generando o son escasas las políticas públicas para la gestión sostenible de nuestro patrimonio natural y cultural, no solamente para el beneficio local sino también para la conservación del ambiente.

$\mathrm{Al}$ igual que los bienes privados, el ambiente enfrenta problemas de escasez relativa dado que tiene usos alternativos, por otro lado, el problema económico se origina en la necesidad de decidir en torno al mejor uso social de los recursos naturales (CUMMINGS, 1986).

La toma de decisiones sobre el uso de los recursos es una tarea que está directamente relacionada con la puesta en valor, en cualquier proceso de toma de decisiones, donde se busca elegir la mejor alternativa para la sociedad. En ese sentido, la teoría económica valora de manera particular los recursos naturales y los servicios ambientales, en particular si se propende al máximo bienestar social con el menor costo posible.

Los ecosistemas proporcionan flujos de bienes y servicios tanto directos como indirectos a los agentes económicos y a la sociedad en general, muchos de estos servicios constituyen un soporte fundamental para la existencia misma de la vida (Ortiz, 2017).

Las ANP en sus distintas categorías, proveen bienes y servicios ambientales escasos, pero fundamental para la sociedad y la economía. Forman parte importante del capital natural, que conservándolo y aprovechándolo inteligentemente podría sustentar nuestra economía en el largo plazo
(Morales León, 2007).

Esto se ve reflejado en el mejoramiento de la calidad de vida y los niveles de bienestar social, es decir capturar las preferencias sociales a preservar ciertos niveles de bienestar logrado por los beneficios generados por el sistema natural o a evitar cierta degradación de la calidad de vida por la pérdida o disminución de sus servicios (Hernandez, 1998).

En el marco del D.S. $\mathrm{N}^{\circ}$ 022-2010-MINAM, el complejo de Choquequirao fue designado como un Área de Conservación Regional (ACR) lo que revela una singular importancia debido a la dualidad natural y cultural que involucra, ya que alberga importantes monumentos arqueológicos Incas y comprende una gran diversidad de ecosistemas, de gran belleza paisajística, y de especies de flora y fauna silvestre, muchas de ellas endémicas (Diario oficial el Peruano, 2010).

\section{Materiales y métodos}

El Área de Conservación Regional Choquequirao-ACRCHOQ, tiene 103 814,39 has, se encuentra ubicada en la margen derecha del río Apurímac, en la geografía de los distritos de Santa Teresa y Vilcabamba, Provincia de La Convención y también de los Distritos de Limatambo y Mollepata, Provincia de Anta del departamento de Cusco. Se ubican también, en las estribaciones de las montañas de la vertiente occidental del nevado Salkantay, rodeado por los nevados de Qoriwayrachina, Sacsarayoc, Choquetarpo, Huamantay y el Padreyoc, albergando al Parque Arqueológico de Choquequirao, a 3100 msnm a media ladera de la montaña que lleva el mismo nombre.

En ACR-CHOQ, presenta espacios naturales que albergan una variedad de zonas de vida relacionadas con los bosques secos, montanos y sub-tropicales, donde 
se dan sucesiones extraordinarias de zonas bioclimáticas y en el que se desarrolla una importante biodiversidad. La ACR CHOQ es una de las 17 ACR que comprende las 167 ANP del SINANPE.

En diciembre del 2010, se crea el ACRCHOQ, con el objetivo de: "conservar la diversidad biológica de los ecosistemas de bosques montanos húmedos, bosques estacionalmente secos, bosques nativos, que albergan numerosas especies endémicas, así como asegurar la conservación del recurso hídrico, los recursos culturales arqueológicos y la continuidad de los procesos biológicos de los ecosistemas presentes en el área".

El ACR CHOQ presenta una alta diversidad específica, principalmente en plantas, como las familias Orchidaceae (orquídeas) con 287 especies y Poaceae con 93 especies también. Se puede observar importantes flujos de bienes y servicios ambientales, de manera cualitativa, entre ellos se tiene los usos para actividades agrícolas y pecuarias (función de producción de alimentos), usos como escenarios para la recreación-turismo e investigación (función de información) y usos de la función de regulación como son los servicios de mantenimiento de clima favorable, drenaje e irrigación natural etc.

Uno de los más destacados usos del ACR-CHOQ, es la provisión de bienes y servicios como escenario cultural y natural, para desarrollar actividades turísticas, en tal sentido se viene implementando, desde hace unos 10 años atrás, como parte de las políticas de promoción turística desplegadas por el MINCETUR, visitas hacia el Parque Arqueológico- $\mathrm{P}^{\circ} \mathrm{A}^{\circ} \mathrm{CHOQ}$ permitiendo un despegue de esta actividad.

El desarrollo del uso turístico y recreativo de las ANP deberá "sujetarse a los objetivos primarios de conservación" de cada una de las áreas, procurando minimizar los impactos ambientales y socioculturales generados, de modo que se logre una actividad turística sostenible" (SINANPE, 1999). Lo anterior indica la preocupación del Estado peruano por el adecuado manejo de los impactos que genera el turismo en las áreas protegidas, preocupación que es recogida por la población y diversos estudiosos del tema.

Una de las instituciones encargadas de administrar el patrimonio cultural del parque arqueológico de Choquequirao es la Dirección Desconcentrada de Cultura de Cusco-DDC y la encargada de instalar e implementar servicios turísticos públicos es el Gobierno Regional de Cusco a través del Proyecto Especial PLAN COPESCO (2017), estas instituciones en estos últimos años han disminuido sus niveles de inversión para implementar infraestructura en materia de turismo.

\section{Resultados y discusión}

\section{Evaluación del flujo turístico a Choquequirao}

En la Tabla 1 se muestra la evolución del flujo turístico hacia Choquequirao entre los años 2002 y 2016 según estadísticas del Ministerio de Comercio Exterior y Turismo (MINCETUR, 2017). En este lapso de tiempo, el flujo turístico ha estado compuesto por turistas nacionales y extranjeros y se ha analizado la evolución por ambos rubros. En estos 15 años, en promedio el $45 \%$ de turistas fueron nacionales y 55\% extranjeros, y en el año 2002 se registraron la visita de 3444 turistas a Choquequirao, habiendo alcanzado el número de visitantes máximo en el año 2008 con 7731 turistas. En el año 2016 se registraron 5993 turistas. En las dos últimas columnas se muestra la evolución de los turistas por orígenes. Se puede percibir que existen períodos de crecimiento como entre los años 2004 a 2007 y períodos de disminución del flujo turístico, como entre los años 2011 a 2013. 
Tabla 1: Flujo de turistas a Choquequirao por origen

\begin{tabular}{|c|c|c|c|c|c|c|c|c|}
\hline Años & $\begin{array}{c}\text { Nacionales } \\
\text { (A) }\end{array}$ & $\begin{array}{c}\text { Extranjeros } \\
\text { (B) }\end{array}$ & Total & $\begin{array}{c}\text { \% turistas } \\
\text { nacionales }\end{array}$ & $\begin{array}{c}\% \text { turistas } \\
\text { extranjeros }\end{array}$ & \begin{tabular}{c|}
$\%$ \\
evolución \\
turistas \\
nacionales \\
\end{tabular} & \begin{tabular}{|c|}
$\%$ \\
evolución \\
turistas \\
extranjeros \\
\end{tabular} & $\begin{array}{c}\% \\
\text { evolución } \\
\text { turistas } \\
\text { totales } \\
\end{array}$ \\
\hline 2002 & 2237 & 1207 & 3444 & $65 \%$ & $35 \%$ & & & \\
\hline 2003 & 1405 & 2145 & 3550 & $40 \%$ & $60 \%$ & $-37 \%$ & $78 \%$ & $3 \%$ \\
\hline 2004 & 3515 & 2313 & 5828 & $60 \%$ & $40 \%$ & $150 \%$ & $8 \%$ & $64 \%$ \\
\hline 2005 & 3996 & 2565 & 6561 & $61 \%$ & $39 \%$ & $14 \%$ & $11 \%$ & $13 \%$ \\
\hline 2006 & 4153 & 2707 & 6860 & $61 \%$ & $39 \%$ & $4 \%$ & $6 \%$ & $5 \%$ \\
\hline 2007 & 4497 & 3205 & 7702 & $58 \%$ & $42 \%$ & $8 \%$ & $18 \%$ & $12 \%$ \\
\hline 2008 & 4389 & 3342 & 7731 & $57 \%$ & $43 \%$ & $-2 \%$ & $4 \%$ & $0 \%$ \\
\hline 2009 & 1732 & 2837 & 4569 & $38 \%$ & $62 \%$ & $-61 \%$ & $-15 \%$ & $-41 \%$ \\
\hline 2010 & 3688 & 3976 & 7664 & $48 \%$ & $52 \%$ & $113 \%$ & $40 \%$ & $68 \%$ \\
\hline 2011 & 2922 & 2861 & 5783 & $51 \%$ & $49 \%$ & $-21 \%$ & $-28 \%$ & $-25 \%$ \\
\hline 2012 & 1047 & 2287 & 3334 & $31 \%$ & $69 \%$ & $-64 \%$ & $-20 \%$ & $-42 \%$ \\
\hline 2013 & 768 & 2878 & 3646 & $21 \%$ & $79 \%$ & $-27 \%$ & $26 \%$ & $9 \%$ \\
\hline 2014 & 1825 & 3043 & 4868 & $37 \%$ & $63 \%$ & $138 \%$ & $6 \%$ & $34 \%$ \\
\hline 2015 & 1255 & 3682 & 4937 & $25 \%$ & $75 \%$ & $-31 \%$ & $21 \%$ & $1 \%$ \\
\hline 2016 & 1299 & 4634 & 5933 & $22 \%$ & $78 \%$ & $4 \%$ & $26 \%$ & $20 \%$ \\
\hline \multicolumn{3}{|c|}{ Fuente: Mincetur (2017) } & Promedio & $45 \%$ & $55 \%$ & & & \\
\hline
\end{tabular}

Entre los años 2002 y 2016 el número de turistas aumentó de 3444 a 5933, es decir en $74 \%$, y en ese mismo período el número de visitantes nacionales disminuyó de 2237 a 1299 , es decir hubo una caída del $42 \%$, mientras que el número de visitantes extranjeros aumentó de 1207 a 4634, es decir hubo un incremento del $283 \%$. En la Tabla 1 se observa que, en los últimos años la participación de turistas extranjeros es mayoritaria. Así en el año 2016 el porcentaje de turistas extranjeros representó el 78\% del total.

\section{Evolución del flujo turístico a Machu- Picchu}

En la Tabla 2 se muestra el flujo turístico hacia Machu-Picchu en el mismo período 2002-2016 pudiéndose verificar lo siguiente: i) en promedio en estos 15 años la participación de turistas extranjeros representó el 69\% del total, mientras que los turistas nacionales el 31\%; ii) entre 2002 y 2016 el número de turistas se incrementó de 457876 a 1'419 507, es decir hubo un incremento del $210 \%$; iii) en este período el flujo de turistas extranjeros se incrementó de 306989 a 996 764, es decir representó un incremento del $225 \%$; en tanto el flujo de turistas nacionales aumentó de 150,887 a 422 743, es decir, aumentó en 181\%.

En el caso del flujo de turistas nacionales se puede ver que ha habido incremento de visitantes, pero en ciertos años como en el 2004, 2008, 2009, 2010, 2014 ha habido también disminución de visitantes; mientras que en el caso de los turistas extranjeros el flujo se ha incrementado de manera regular a excepción de los años 2010 y 2014.

\section{Comparación entre flujo turístico en Machu-Picchu y Choquequirao}

En la Región Cusco el principal atractivo viene a ser el Santuario Histórico de Machupicchu, que genera el $98 \%$ de los ingresos del sector turismo, mientras que el ACR Choquequirao es un producto turístico que viene posicionándose en el mercado de forma lenta, pese a contar con una riqueza natural y cultural similar a la de Machupicchu, razón por la que se conoce a Choquequirao como la hermana de Machupicchu. 
Tabla 2. Flujo de turistas a Machu Picchu por origen

\begin{tabular}{|c|c|c|c|c|c|c|c|c|}
\hline Años & $\begin{array}{c}\text { Nacionales } \\
\text { (A) }\end{array}$ & $\begin{array}{c}\text { Extranjeros } \\
\text { (B) }\end{array}$ & Total & $\begin{array}{c}\% \text { turistas } \\
\text { nacionales }\end{array}$ & $\begin{array}{c}\% \text { turistas } \\
\text { extranjeros }\end{array}$ & \begin{tabular}{c|}
$\%$ \\
evolución \\
turistas \\
nacionales \\
\end{tabular} & \begin{tabular}{|c|}
$\%$ \\
evolución \\
turistas \\
extranjeros \\
\end{tabular} & $\begin{array}{c}\% \\
\text { evolución } \\
\text { turistas } \\
\text { totales } \\
\end{array}$ \\
\hline 2002 & 150,887 & 306,989 & 457,876 & $33 \%$ & $67 \%$ & & & \\
\hline 2003 & 190,003 & 333,805 & 523,808 & $36 \%$ & $64 \%$ & $26 \%$ & $9 \%$ & $14 \%$ \\
\hline 2004 & 184,239 & 404,199 & 588,438 & $31 \%$ & $69 \%$ & $-3 \%$ & $21 \%$ & $12 \%$ \\
\hline 2005 & 204,638 & 475,315 & 679,953 & $30 \%$ & $70 \%$ & $11 \%$ & $18 \%$ & $16 \%$ \\
\hline 2006 & 210,882 & 480,741 & 691,623 & $30 \%$ & $70 \%$ & $3 \%$ & $1 \%$ & $2 \%$ \\
\hline 2007 & 251,990 & 548,168 & 800,158 & $31 \%$ & $69 \%$ & $19 \%$ & $14 \%$ & $16 \%$ \\
\hline 2008 & 242,101 & 616,115 & 858,216 & $28 \%$ & $72 \%$ & $-4 \%$ & $12 \%$ & $7 \%$ \\
\hline 2009 & 233,388 & 581,880 & 815,268 & $29 \%$ & $71 \%$ & $-4 \%$ & $-6 \%$ & $-5 \%$ \\
\hline 2010 & 227,089 & 472,742 & 699,831 & $32 \%$ & $68 \%$ & $-3 \%$ & $-19 \%$ & $-14 \%$ \\
\hline 2011 & 300,683 & 670,959 & 971,642 & $31 \%$ & $69 \%$ & $32 \%$ & $42 \%$ & $39 \%$ \\
\hline 2012 & 351,965 & 762,469 & $1,114,434$ & $32 \%$ & $68 \%$ & $17 \%$ & $14 \%$ & $15 \%$ \\
\hline 2013 & 372,960 & 804,348 & $1,177,308$ & $32 \%$ & $68 \%$ & $6 \%$ & $5 \%$ & $6 \%$ \\
\hline 2014 & 298,986 & 842,191 & $1,141,177$ & $26 \%$ & $74 \%$ & $-20 \%$ & $5 \%$ & $-3 \%$ \\
\hline 2015 & 371,462 & 911,053 & $1,282,515$ & $29 \%$ & $71 \%$ & $24 \%$ & $8 \%$ & $12 \%$ \\
\hline 2016 & 422,743 & 996,764 & $1,419,507$ & $30 \%$ & $70 \%$ & $14 \%$ & $9 \%$ & $11 \%$ \\
\hline \multicolumn{3}{|c|}{ Fuente: Mincetur (2017) } & Promedio & $31 \%$ & $69 \%$ & & & \\
\hline
\end{tabular}

De acuerdo al perfil del Turista Extranjero (PROMPERU, 2013), da a conocer que Machu Picchu siguió siendo la razón más importante para visitar el Perú (73\% del total de menciones). Destacaron por esta preferencia los turistas procedentes de Japón, Australia y Canadá. Y de acuerdo a este perfil, se puede observar que el 19\% de turistas extranjeros tienen preferencia por desarrollar caminatas-trekking y $29 \%$ por conocer áreas naturales protegidas, y $43 \%$ por visitar sitios arqueológicos, siendo estos parámetros importantes para promocionar Choquequirao por sus atributos culturales y de naturaleza.

En la Tabla 3 se muestra una comparación entre el número de visitantes a MachuPicchu y a Choquequirao, pudiéndose establecer que el flujo de visitantes hacia Choquequirao representó en los últimos 15 años, menos del $1 \%$ del flujo de visitantes hacia Machu-Picchu.

\section{Perspectivas de la puesta en valor de Choquequirao}

\section{Descripción de la problemática económica de Choquequirao}

Actualmente, las instalaciones turísticas en el ACR Choquequirao son precarias, con señalización inadecuada, accesos peatonales al parque en condiciones críticas de seguridad y mantenimiento, escasos espacios para que los turistas puedan descansar y observar el paisaje, inexistencia de centros de información y control a lo largo de las rutas de acceso.

En las rutas de acceso, los turistas no cuentan con un mobiliario adecuado, como barandas, basureros y servicios higiénicos, estos servicios solo se pueden encontrar al ingresar al parque en el sector denominado de Sunchupata y en este lugar también se ubica la boletería administrada por la Dirección Desconcentrada de Cultura del CuscoDDC. En la Tabla 5 se muestran las tarifas de la entrada al Complejo Arqueológico de Choquequirao, que ha sido reajustada para este año 2017 según la Resolución 030-2016 del Ministerio de Cultura; en el caso de la tarifa para el público en general ha habido un incremento de $\mathrm{S} / 55$ a S/ 60, mientras que en el caso de la tarifa para estudiantes se mantiene igual en $\mathrm{S} / 30$, y la tarifa especial ha aumentado de S/ 15 a S/ 25. 
Tabla 3. Comparación de visitantes Choquequirao y Machu Picchu

\begin{tabular}{|c|c|c|c|c|c|}
\hline Años & $\begin{array}{c}\text { Visitantes } \\
\text { Choquequirao }\end{array}$ & $\begin{array}{l}\text { Visitantes } \\
\text { Machu- } \\
\text { Picchu }\end{array}$ & $\begin{array}{l}\text { Total visitantes } \\
\text { Choquequirao y } \\
\text { Machu-Picchu }\end{array}$ & $\begin{array}{c}\% \\
\text { Choquequirao }\end{array}$ & $\begin{array}{c}\% \\
\text { Machupicchu }\end{array}$ \\
\hline 2002 & 3,444 & 457,876 & 461,320 & $0.7 \%$ & $99.3 \%$ \\
\hline 2003 & 3,550 & 523,808 & 527,358 & $0.7 \%$ & $99.3 \%$ \\
\hline 2004 & 5,828 & 588,438 & 594,266 & $1.0 \%$ & $99.0 \%$ \\
\hline 2005 & 6,561 & 679,953 & 686,514 & $1.0 \%$ & $99.0 \%$ \\
\hline 2006 & 6,860 & 691,623 & 698,483 & $1.0 \%$ & $99.0 \%$ \\
\hline 2007 & 7,702 & 800,158 & 807,860 & $1.0 \%$ & $99.0 \%$ \\
\hline 2008 & 7,731 & 858,216 & 865,947 & $0.9 \%$ & $99.1 \%$ \\
\hline 2009 & 4,569 & 815,268 & 819,837 & $0.6 \%$ & $99.4 \%$ \\
\hline 2010 & 7,664 & 699,831 & 707,495 & $1.1 \%$ & $98.9 \%$ \\
\hline 2011 & 5,783 & 971,642 & 977,425 & $0.6 \%$ & $99.4 \%$ \\
\hline 2012 & 3,334 & $1,114,434$ & $1,117,768$ & $0.3 \%$ & $99.7 \%$ \\
\hline 2013 & 3,646 & $1,177,308$ & $1,180,954$ & $0.3 \%$ & $99.7 \%$ \\
\hline 2014 & 4,868 & $1,141,177$ & $1,146,045$ & $0.4 \%$ & $99.6 \%$ \\
\hline 2015 & 4,937 & $1,282,515$ & $1,287,452$ & $0.4 \%$ & $99.6 \%$ \\
\hline 2016 & 5,933 & $1,419,507$ & $1,425,440$ & $0.4 \%$ & $99.6 \%$ \\
\hline
\end{tabular}

Tabla 4. Rutas turísticas hacia el complejo Choquequirao

\begin{tabular}{|c|l|c|}
\hline Rutas & \multicolumn{1}{|c|}{ Tramos } & Distancia \\
\hline 1 & Cachora-Choquequirao & $30 \mathrm{~km}$ \\
\hline 2 & Huanipaca-Tambobamba-Choquequirao & $17 \mathrm{~km}$ \\
\hline 3 & Huancacalle - Yanama-Choquequirao & $68 \mathrm{~km}$ \\
\hline 4 & Santa Teresa - Qollpapampa-Totora-Yanama-Choquequirao & $70 \mathrm{~km}$ \\
\hline 5 & Hidroeléctrica Machu-Picchu-Lucmabamba-Choquequirao & $74 \mathrm{~km}$ \\
\hline 6 & Mollepata-Salquantay - Qollpampa-Choquequirao & $106 \mathrm{~km}$ \\
\hline \multicolumn{2}{|l}{ Fuente: Plan Maestro de Choquequirao, DDC 2000 } \\
\hline
\end{tabular}

Tabla 5. Tarifa de la entrada a Choquequirao $($ En S/)

\begin{tabular}{|c|c|c|}
\hline Publico & Tarifa 2016 & Tarifa 2017 \\
\hline \begin{tabular}{l|l|}
1 & Adultos en general \\
\end{tabular} & 55 & 60 \\
\hline $\begin{array}{l}\text { Estudiantes de educación } \\
\text { superior universitaria yno- } \\
2 \text { universitaria }\end{array}$ & 30 & 30 \\
\hline $3 \mid$ Entrada Especial $^{*}$ & 15 & 25 \\
\hline \multicolumn{3}{|c|}{ Fuente: Ministerio de Cultura. R.M. 030-2016. } \\
\hline
\end{tabular}

La DDC, es el encargado además de mantener los caminos de acceso y el propio parque, con recurso financieros y humanos insuficientes; actualmente cuenta con un jefe de área, dos asistentes y 15 conservadores, y las tarifas que se recaudan ingresan a una bolsa única de la DDC y luego es distribuido por el Ministerio de Cultura a nivel de todo el país y le asignan el presupuesto para las actividades operativas y de mantenimiento del Complejo de Choquequirao, que generalmente son subvencionadas por los ingresos generados por Machupicchu; es así que para el año 2015 tuvieron un presupuesto asignado de solo S/ 649 000, pero lo que realmente necesitan es $\mathrm{S} / 1^{\prime} 235000 .^{1}$

${ }^{1}$ Plan Operativo Institucional de Choquequirao de la 
Entre una de las actividades de mantenimiento que desarrollan los 15 conservadores de la DDC, es acopiar los residuos sólidos producidos por los turistas a lo largo de las rutas de acceso, especialmente en la Ruta 1, en las demás rutas no se desarrollan, son evacuadas en lugares inaccesibles, bosques marginales o en los barrancos de algunos tramos de las rutas de acceso. Estos residuos sólidos están en crecimiento de acuerdo al número de turistas que ingresan al parque, es así que datos registrados por un estudio desarrollado por DDC, la tendencia de crecimiento de los residuos sólidos superará los $1500 \mathrm{~kg}$. al año, solo considerando las principales rutas. En la Tabla 6 se muestran las principales rutas ofrecidas por las agencias por las agencias turísticas para visitar Choquequirao.

De acuerdo al diagnóstico desarrollado por la DCC, indica con respecto a la conservación y mantenimiento del parque arqueológico (Tabla 7).

Estas dificultades y problemas han venido limitando el acceso y el incremento del número de visitantes hacia Choquequirao, a pesar de los atractivos turísticos, culturales y ecológicos que presenta el complejo.

\section{Inversiones a realizarse para mejorar la conectividad hacia Choquequirao}

Siendo la segunda ciudadela inca importante de la región Cusco que permitiría sumar un nuevo atractivo a los visitantes, que hoy tienen a Machu Picchu como principal motivo para llegar hasta esta zona de Perú. Claro, Machu Picchu ya no puede crecer mucho más en cuanto a la cantidad de visitantes, y si las autoridades peruanas quieren seguir impulsando el crecimiento del turismo en el país tendrán que lograr, entre otras cosas, que los viajeros se queden más días y tengan más lugares para visitar. Una parte importante de Choquequirao ya ha sido excavada y está abierta para la visita de los turistas desde hace un buen tiempo.

Hoy visitar Choquequirao toma unos 5 días, debido a que la caminata hasta la ciudadela inca es de 2 días, hay que realizar una caminata en la montaña, que es físicamente exigente. El resultado es que al menos por ahora la cantidad de turistas en Choquequirao es muy baja, y que claramente, si se quiere insertar al lugar dentro de las rutas del turismo masivo, se tendría que facilitar el acceso.

El proyecto del teleférico cuesta 62 millones de dólares, y tiene una extensión de $5,4 \mathrm{~km}$ : el primer tramo proyectado con una extensión de $3,5 \mathrm{~km}$ y el segundo tramo con una extensión de 1,9 km. Con esta infraestructura se reduciría el tiempo de viaje hasta Choquequirao de 2 días de ida y 2 días de vuelta, a tan solo 15 minutos de ida y 15 minutos de vuelta.

El gobierno adicionalmente a este proyecto debería realizar gastos de mantenimiento, en lo que se refiere a limpieza de caminos, así como implementar programas y proyectos de restauración de áreas deterioradas, y actividades de investigación científica sobre los ecosistemas del ACR, los nevados, la flora y fauna local. Esta inversión es necesario ya que el parque arqueologico no registra mejoras en los distintos recintos por los que está compuesto, y no se manifiesta en la puesta en valor de las nueve zonas en la que está clasificada el parque.

De acuerdo al Banco de Inversiones que maneja el MEF, se encontró proyectos que esperan ser ejecutados y obviamente precisan del financiamiento por el Estado, y como no lo ven como prioridad año a año se deja de contar con dicho presupuesto. Para proyectos que precisan mejoramiento en los servicios turísticos públicos en CHOQ, se estimó una inversión de S/ 80’945,148.00 y para proyectos en temas de conservación del área natural protegida S/ 49'294,749 (Tabla 8). 
Tabla 6. Circuitos turísticos ofrecidos en Choquequirao por las agencias de viaje

\begin{tabular}{|l|c|c|}
\hline \multicolumn{1}{|c|}{ Circuito turístico } & Tiempo recorrido & Escala \\
\hline $\begin{array}{l}\text { Andenes de Cultivo-Plaza Principal-Cancha de } \\
\text { Camélidas - Usno-Vivienda de Sacerdotes - } \\
\text { Viviendas populares - Plaza Principal }\end{array}$ & 1 hora 30 minutos & Corto \\
\hline $\begin{array}{l}\text { Andenes de Cultivo-Plaza Principal-Sector } \\
\text { Hanan - Sector Llamas - Plaza Principal - } \\
\text { Cancha para Camélidos - Usno - Vivienda } \\
\text { Sacerdotes - Viviendas Populares }\end{array}$ & & \\
\hline $\begin{array}{l}\text { Andenes de Cultivo- Plaza Principal - Cancha } \\
\text { para camélidos - Usno - Vivienda Sacerdotes - } \\
\text { Viviendas Populares - Andenes de Cultivo - } \\
\text { Pinchaunuyoc - Plaza Principal }\end{array}$ & & \\
\hline Fuente: Dircetur, 2012 & & Largo \\
\hline
\end{tabular}

Tabla 7. Brechas en la conservación y mantenimiento del Complejo de Choquequirao

\begin{tabular}{|c|c|}
\hline 1 & $\begin{array}{l}\text { Los trabajos de conservación preventiva no son las adecuadas, sobre los muros se observa la } \\
\text { existencia de plantas herbáceas que están originando el desprendimiento de revoque o estuco } \\
\text { original del edificio }\end{array}$ \\
\hline 2 & $\begin{array}{l}\text { Sobre los elementos líticos se ha observado la eflorescencia de sulfato de calcio como } \\
\text { consecuencia de la aplicación de cemento y cal en su intervención este material está originando } \\
\text { la exfoliación de muchos elementos líticos, estas han emergido en la base de las piedras en } \\
\text { forma de cristales de tipo estalactita }\end{array}$ \\
\hline 3 & $\begin{array}{l}\text { Por estar edificado la plaza principal sobre un antiguo relleno, por los últimos movimientos } \\
\text { geodinámicas es imprescindible desarrollar estudios sobre la geodinámica de suelos, los } \\
\text { deslizamientos alrededor del monumento podrían estar afectando de forma comprometida la } \\
\text { estabilidad del monumento }\end{array}$ \\
\hline 4 & $\begin{array}{l}\text { La filtración de las aguas pluviales se deposita en la base de los muros principales, estas afectan } \\
\text { la estabilidad de los sectores más importante de Choquequirao }\end{array}$ \\
\hline 5 & $\begin{array}{l}\text { La falta de mantenimiento permanente hace que las evidencias de los enlucidos de reposición } \\
\text { y revoques originales, vienen perdiendo estabilidad }\end{array}$ \\
\hline 6 & $\begin{array}{l}\text { Los revoques originales y aquellas que han sido recompuestas sufren agresión directa por parte } \\
\text { de los visitantes que dejan grafitis con color o en bajo relieve que ocasiona daños sobre el } \\
\text { monumento. }\end{array}$ \\
\hline 7 & $\begin{array}{l}\text { Falta realizar estudios arquitectónicos de los sectores para poder efectuar una mejor } \\
\text { determinación sobre su función, asociación cultural y su cronológica }\end{array}$ \\
\hline 8 & $\begin{array}{l}\text { Falta realizar excavaciones arqueológicas para tener un mejor estudio de la arquitectura, estas } \\
\text { excavaciones se pueden aprovechar para poder determinar algunas causas internas que pueda } \\
\text { estar afectando la integridad de patrimonio cultural }\end{array}$ \\
\hline 9 & $\begin{array}{l}\text { Los espacios exteriores del muro circundante, en la plataforma ceremonial del Usnho, se vienen } \\
\text { utilizando para la acumulación de basura y desechos sólidos, así mismo parte del acceso es } \\
\text { utilizado como un mirador hacia la plaza principal, lo que propicia que los turistas se posen } \\
\text { sobre los muros para la toma de fotografías, pudiendo ocasionar graves daños en el patrimonio. }\end{array}$ \\
\hline 10 & $\begin{array}{l}\text { El sistema de canales de desfogue de lluvia han colapsado, produciendo aniegos de las estructuras, } \\
\text { estas arrastran sales solubles directamente hacia la base de los muros, por lo que urgen efectuar } \\
\text { tratamientos integrales para la preservación de este importante resto arquitectónico. }\end{array}$ \\
\hline 11 & $\begin{array}{l}\text { Los trabajos en excavación arqueológica en promedio en el parque reportan un avance de } 20 \% \\
\text { y un } 80 \% \text { de área faltante }\end{array}$ \\
\hline
\end{tabular}

Fuente: Diagnóstico de la Dirección de Cultura de Cusco (2015) 
Tabla 8. Presupuesto para turismo y conservación en Choquequirao

\begin{tabular}{|l|r|r|r||}
\hline Proyectos de inversión & En Soles S/.En Dólares (US \$) & \multicolumn{1}{c|}{$\%$} \\
\hline Turismo & $31^{\prime} 650,399$ & $9^{\prime} 174,029$ & $39 \%$ \\
\hline Conservación & $49^{\prime} 294,749$ & $14^{\prime} 288,333$ & $61 \%$ \\
\hline Conservación y Turismo & $80^{\prime} 945,148$ & $23^{\prime} 462,362$ & $100 \%$ \\
\hline
\end{tabular}

Fuente: Banco de Proyectos. SNIP.Dirección de Políticas de Inversión del Ministerio de Economía

Diciembre de 2015. TC Octubre 2016= US $\$ 1=S / 3.45$

\section{El caso del teleférico de Chachapoyas y comparación con Choquequirao}

Una iniciativa similar fue ejecutada en Kuelap, en Chachapoyas. Los resultados, al menos en los números, son positivos para esta última experiencia, a pesar que el gran tema de Chachapoyas, sigue siendo en todo caso la conectividad aérea.

En la Tabla 9 se muestra la comparación entre marzo de 2016 y marzo de 2017, esta última fecha correspondiente a cuando entró en operación el teleférico de Kuelpa. Entre marzo y octubre de 2016, comparado con el mismo período entre marzo y octubre de 2017, se ha incrementado el flujo de visitantes en Kuelap de 36531 a 88578 , es decir más 52000 visitantes más, lo cual representa un incremento significativo.

Tabla 9. PIncremento del flujo de visitantes 2016-2017 en Kuelap por la implementación del teléfono

\begin{tabular}{|l|r|r|r|}
\hline \multicolumn{1}{|c|}{ Meses } & \multicolumn{1}{c|}{$\mathbf{2 0 1 6}$} & \multicolumn{1}{c|}{$\mathbf{2 0 1 7}$} & \multicolumn{1}{c|}{$\%$} \\
\hline Marzo & 3009 & 5412 & $80 \%$ \\
\hline Abril & 1462 & 8130 & $456 \%$ \\
\hline Mayo & 2829 & 6883 & $143 \%$ \\
\hline Junio & 2593 & 9001 & $247 \%$ \\
\hline Julio & 8240 & 18058 & $119 \%$ \\
\hline Agosto & 6979 & 18986 & $172 \%$ \\
\hline Septiembre & 5094 & 10731 & $111 \%$ \\
\hline Octubre & 6325 & 11377 & $80 \%$ \\
\hline Total & 36531 & 88578 & $142 \%$ \\
\hline Fuente: MINCETUR. Eaboración propia \\
\hline
\end{tabular}

En la Tabla 10 se muestra el flujo de visitantes a Kuelap, que fundamentalmente está compuesto por visitantes nacionales, a diferencia de Choquequirao que es visitado principalmente por visitantes extranjeros.

El crecimiento de la actividad turística en Choquequirao no es un hecho aislado de la realidad nacional, ya que el turismo en el Perú ha experimentado un crecimiento sostenido desde el año 2002. Así, esta iniciativa del teleférico planea incrementar el número de turistas nacionales e internacionales de 5500 visitantes anualmente a más de 100 000 visitantes durante el primer año de operaciones.

La puesta en valor de Choquequirao por tanto generaría un importante efecto multiplicador en la economía local donde se ubica el atractivo turístico y al desarrollarse la actividad turística se forja una serie de impactos, entre los principales impactos positivos se puede mencionar su contribución a la generación de empleos; empleo directo (hoteles, restaurantes, agencias de viaje), empleo indirecto (taxistas, recepcionistas, mozos, comunidades campesinas). Es importante, también considerar su conexión con otros sectores empresariales, como construcción, comercio y servicios en general.

\section{La puesta en valor en las áreas protegidas: aspectos conceptuales}

Las estrategias más frecuentes que se aplican para lograr un turismo sustentable son 
la creación de áreas naturales protegidas (ANP) y de zonas arqueológicas (ZA), que se complementan con la educación o interpretación ambiental, la promoción del bienestar de las comunidades locales, la conservación de la naturaleza (Quadri, 2006; Garayo, 2001; Halffter, 2011) y de la herencia cultural, por lo cual apoyan el desarrollo sustentable.

Sin embargo, la creación de estos sitios no asegura la integridad ecológica o cultural, sino que incluso podría incrementar los daños debido al uso turístico, por lo cual se requiere de estrategias concretas bajo los principios del desarrollo sustentable, para asegurar la conservación de la naturaleza y la herencia cultural que estos espacios albergan, apoyar a las comunidades receptoras, facilitar el disfrute y evitar posibles impactos negativos de los visitantes y obtener beneficios económicos (Romano \& Serrano, 2013).

Lorenzo (1989) y Trigger (1989) afirman que la creación de ANP y ZA son parte de una estrategia de creación de valores identitarios o turísticos, en cuyo caso se impulsa la comercialización para generar beneficios económicos por medio de su puesta en valor como atractivo turístico con base económica, y no cultural o natural (Moreno, 2004), aunque probablemente el mejor argumento a favor del uso turístico de ANP y ZA es la función formativa o educativa, ya que al permitir el acceso de los visitantes se fomenta la conservación y preservación de estas áreas y también de otros espacios urbanos o rurales similares.

Así, el turismo mal administrado junto con la masificación de las visitas, conlleva a impactos negativos para estos sitios, que puede causar daños irreparables a la biodiversidad, suelo y agua (Williams \& Ponsford, 2009) o a los monumentos históricos, lo cual podría interferir con otros beneficios que deben brindar dichas áreas. Existen amplias referencias sobre impactos negativos que el turismo provoca a monumentos, áreas de vegetación o

Tabla 10. Flujo de turistas a Kuelap (Amazonas) por origen

\begin{tabular}{|c|r|r|r|r|r|r|r|r|}
\hline Años & $\begin{array}{c}\text { Nacionales } \\
\text { (A) }\end{array}$ & $\begin{array}{r}\text { Extranjeros } \\
\text { (B) }\end{array}$ & Total & $\begin{array}{c}\text { \% turistas } \\
\text { nacionales }\end{array}$ & $\begin{array}{c}\text { \% turistas } \\
\text { extranjeros }\end{array}$ & $\begin{array}{c}\% \\
\text { evolución } \\
\text { turistas } \\
\text { nacionales }\end{array}$ & $\begin{array}{c}\% \\
\text { evolución } \\
\text { turistas } \\
\text { extranjeros }\end{array}$ & $\begin{array}{c}\% \\
\text { evolución } \\
\text { turistas } \\
\text { totales }\end{array}$ \\
\hline 2002 & 7,471 & 1,466 & 8,937 & $84 \%$ & $16 \%$ & & & $15 \%$ \\
\hline 2003 & 8,129 & 1,687 & 9,816 & $83 \%$ & $17 \%$ & $9 \%$ & $10 \%$ \\
\hline 2004 & 8,391 & 1,683 & 10,074 & $83 \%$ & $17 \%$ & $3 \%$ & $0 \%$ & $3 \%$ \\
\hline 2005 & 7,223 & 2,250 & 9,473 & $76 \%$ & $24 \%$ & $-14 \%$ & $34 \%$ & $-6 \%$ \\
\hline 2006 & 8,651 & 3,334 & 11,985 & $72 \%$ & $28 \%$ & $20 \%$ & $48 \%$ & $27 \%$ \\
\hline 2007 & 10,462 & 4,241 & 14,703 & $71 \%$ & $29 \%$ & $21 \%$ & $27 \%$ & $23 \%$ \\
\hline 2008 & 12,003 & 5,393 & 17,396 & $69 \%$ & $31 \%$ & $15 \%$ & $27 \%$ & $18 \%$ \\
\hline 2009 & 13,436 & 5,106 & 18,542 & $72 \%$ & $28 \%$ & $12 \%$ & $-5 \%$ & $7 \%$ \\
\hline 2010 & 17,541 & 6,155 & 23,696 & $74 \%$ & $26 \%$ & $31 \%$ & $21 \%$ & $28 \%$ \\
\hline 2011 & 23,055 & 6,376 & 29,431 & $78 \%$ & $22 \%$ & $31 \%$ & $4 \%$ & $24 \%$ \\
\hline 2012 & 21,905 & 6,055 & 27,960 & $78 \%$ & $22 \%$ & $-5 \%$ & $-5 \%$ & $-5 \%$ \\
\hline 2013 & 27,195 & 6,300 & 33,495 & $81 \%$ & $19 \%$ & $24 \%$ & $4 \%$ & $20 \%$ \\
\hline 2014 & 33,042 & 7,104 & 40,146 & $82 \%$ & $18 \%$ & $22 \%$ & $13 \%$ & $20 \%$ \\
\hline 2015 & 29,092 & 7,293 & 36,385 & $80 \%$ & $20 \%$ & $-12 \%$ & $3 \%$ & $-9 \%$ \\
\hline 2016 & 45,397 & 10,613 & 56,010 & $81 \%$ & $19 \%$ & $56 \%$ & $46 \%$ & $54 \%$ \\
\hline $2017^{*}$ & 69,045 & 15,111 & 84,156 & $82 \%$ & $18 \%$ & $52 \%$ & $42 \%$ & $50 \%$ \\
\hline Fuente: Mincetur 2017$).{ }^{*}$ Enero a Octubre 2017 & Promedio & $78 \%$ & $22 \%$ & & & \\
\hline
\end{tabular}


recreación (Newsome et al., 2002; Buckley, 2004; Pickering \& Hill, 2007), o como causante de conflictos sociales (Kuvan \& Akan, 2005; Kousis, 1989).

La UNESCO en su programa "El hombre y la Biósfera", promovió un cambio significativo en la política de conservación de las ANP, integrando la participación social como estrategia para el manejo sustentable, que aseguraría los servicios ambientales a la vez de beneficiar a sus pobladores (Halffter, 2011). El modelo favorece la administración adaptativa o compartida (Límites de Cambio Aceptable) en las ANP y promueve la participación activa de las comunidades locales para alcanzar los objetivos de conservación y regresar dicho apoyo en la forma de beneficios sociales y económicos (Clark \& Clarke, 2011; García et al., 2011; Glaser et al., 2010). Sin embargo, este modelo no está exento de críticas, ya que así como los turistas son una amenaza para la conservación, también las poblaciones locales pueden ser una fuente de riesgo (Coppock, 1982). En las ZA, para contrarrestar las amenazas de turistas o residentes, tradicionalmente se ha aplicado un modelo centralizado de preservación tipo "comando y control" (Capacidad de Carga Turística), caracterizado por ser coercitivo, vertical, centralizado, y no considerar el entorno social, vinculado a la marginalización de las poblaciones pobres (Luttrel et al., 2007; Wunder, 2005) por el enfoque de exclusión y alienación, en el cual los residentes son considerados el problema que hace insustentable la puesta en valor del patrimonio.

Según la Ley General del Patrimonio Cultural de la Nación, Ley 28296 (Diario Oficial el Peruano, 2004), los sitios arqueológicos como Choquequirao en Cusco, se encuentran enmarcados dentro de la categoría específica de patrimonio cultural material arqueológico inmueble. Cada uno de estos términos indica la naturaleza histórica, origen y características del bien patrimonial así como las posibilidades y limitantes legales respecto a su protección, gestión y aprovechamiento. En concordancia con esta ley se encuentran otras normas importantes que especifican y detallan cómo se deben desarrollar las acciones antes mencionadas, las más importantes son el Reglamento de la Ley (DS 011-2006-ED) y el Reglamento de Intervenciones Arqueológicas, DS 0032014-MC (Diario Oficial el Peruano, 2014).

Según las especificaciones correspondientes a Choquequirao que cuenta con resolución de reconocimiento como parque arqueológico, antes de la realización de cualquier intervención de acondicionamiento de infraestructura para visitantes, debe realizarse en primera instancia un Proyecto de Investigación Arqueológica para determinar las aproximaciones iniciales al conocimiento de la historia del sitio, en segunda instancia o de manera paralela a la investigación, debe darse el proyecto de conservación y/o restauración de la edificación para evitar que las estructuras más debilitadas constituyan un riesgo tanto para el visitante como para la integridad del monumento.

Posteriormente y tomando como base la información preliminar resultado de la investigación, principalmente aquella información donde se detalla el inventario y ubicación total de estructuras de carácter arqueológico, se procede a elaborar el proyecto de puesta en valor, que el concepto actual que se maneja en manuales y guías corresponde a la continuación de los trabajos de investigación y conservación así como la adición de infraestructura que viabilice el turismo y la entrada al monumento a visitantes externos. Este proyecto debe considerar el tamaño y de las características de los sectores del monumento que pueden ser abiertos al público para establecer la capacidad de carga. A medida de que el monumento tenga mayores áreas investigadas, conservadas 
y acondicionadas puede aumentar progresivamente la capacidad de carga. Las estructuras que se construyan deben ir de acuerdo a las características arquitectónicas del monumento, no deben romper con la armonía del paisaje, deben estar construidos con materiales que no signifiquen la destrucción o afectación de otras estructuras arqueológicas.

En el caso de que se requiera la construcción de accesos e infraestructura con material noble, que implique maquinaria pesada o movimiento de tierras, las normas mencionadas indican que sobre el trazo de dichas construcciones debe realizarse: 1) Un proyecto de evaluación arqueológica, para determinar la existencia o inexistencia de restos arqueológicos; En caso de que la evaluación no encuentre restos en superficie 2) Contar con un CIRA (certificado de inexistencia de restos arqueológicos) emitido por la Dirección Desconcentrada de Cultura de Cusco y comprometerse a realizar la obra civil con un arqueólogo monitor que verifique en campo que la obra no está destruyendo patrimonio escondido bajo superficie; En caso de que la evaluación sí encuentre restos arqueológicos 3) Elaborar y proponer ante el Ministerio de Cultura un Proyecto de Rescate Arqueológico, donde la entidad o empresa que se esté responsabilizando de la construcción retire -con arqueólogos profesionales y debidamente acreditados con número de Registro Nacional de Arqueólogos y/o Consultora en Arqueología acreditada - todo el material arqueológico que se encuentre en riesgo producto del movimiento de tierras. 4) Se procede a realizar la obra siempre bajo el criterio de que la afectación al paisaje debe ser la menor posible.

Durante los primeros años de implementación de las leyes referentes al Patrimonio Cultural de la Nación se empleó de manera sucesiva términos como "gestión cultural", "gestión del patrimonio" y "puesta en valor". El primero hasta la fecha es ampliamente usado y se refiera a la gestión en todos los ámbitos culturales, tales como la música, el cine, arte en general y temas histórico-antropológicos. En el caso del segundo, también muy usado hasta la fecha, se refiere de manera más específica a la herencia cultural que poseemos como país que puede darse a nivel del patrimonio cultural material (una huaca, una momia, una iglesia colonial, un documento histórico, etc.) o a nivel de patrimonio cultural inmaterial (las danzas, la recetas gastronómicas, música del folklore, lenguas nativas, entre otros). La puesta en valor sin embargo, es un término que fue muy usado pero que ya está siendo superado por otros términos como "puesta en uso público" o "puesta en uso social".

Se va dejando de lado de manera paulatina porque solamente consideraba que el monumento debía ser investigado, conservado y explotado por parte del Estado de manera restringida y exclusiva, sin considerar que existen diversos usos potenciales tanto por parte de la población, como por parte de entidades académicas, otras entidades del estado y finalmente por la empresa privada. La corriente de cambio en la terminología de los proyectos también responde a los diversos documentos emitidos por entidades internacionales (UNESCO, ICCOMOS, ICCROM, PNUD) que promueven que los Estados involucren a la mayor cantidad posible de actores sociales en los proyectos de conservación, promoción y aprovechamiento de los sitios considerados como Patrimonio Cultural de la Nación o Patrimonio Cultural Mundial. Según todas estas corrientes de gestión se debe desarrollar capacidades locales de autogestión, de manera que después del primer "empujón" por parte del Estado luego de un tiempo el proyecto se vuelva sustentable y sostenible a todo nivel. UNESCO propone mucho el uso de "puesta en uso público", que le da mucho 
énfasis a la participación de la empresa privada y a la participación de la población local para el desarrollo de capacidades, tanto mejor si como producto del desarrollo turístico es la misma población local la que empieza a generar empresas comunitarias o locales. El Ministerio de Cultura del Perú, viene tomando como referencia este término en una versión de gestión cultural muy propia de la realidad peruana, que es la "puesta en uso social". Esta corriente de gestión, comprende los componentes de conservación e investigación de la puesta en valor, pero también considera la participación activa de la población para la conservación y aprovechamiento del monumento, así como la apertura del mismo para la conservación de los valores culturales y religiosos propios de la zona y la apertura a la educación de los niños y población local en general. La puesta en uso social comprende los siguientes usos por parte de las comunidades, entidades gubernamentales y académicas, y empresa privada: a) Uso investigación, científico y educativo; b) Uso comunitario (antropológicas y sociales); c) Uso recreativo y actividades económicas.

Sin embargo y pese a que este nuevo concepto abarca todos los tipos de valor potencial de un sitio arqueológico, la normativa nacional con la que contamos tiene muchas inconsistencias, limitaciones y vacíos que no permiten ponerlo en práctica de manera fluida y eficiente, y esta es una realidad con la que la propia entidad rectora (Ministerio de Cultura, 2016) se choca a diario para la conformación de sus propios proyectos de gestión.

\section{Conclusiones}

Dentro de los principales beneficios es indiscutiblemente la dinamización de la economía, la conservación e investigación del lugar y la apertura al Perú y al mundo de un sitio monumental producto de nuestra herencia ancestral. Los conocimientos producidos hasta la fecha no son difundidos de manera masiva, sin embargo la construcción de facilidades y accesos para los visitantes externos facilita la difusión de todo precedente arqueológico.

Dentro de las contraproducencias es que el aprovechamiento económico del sitio podría no verse explotado principalmente por las comunidades sino por otros operadores turísticos de mayores capitales que se lleven las ganancias sin que estas sean reinvertidas de manera local ya sea en nuevos negocios o en pagos de honorarios locales.

Uno de los grandes vacíos en el marco legal del Patrimonio Cultural es el que deja la gestión ambiental de los monumentos. Si bien los planes maestros, planes de uso social o puesta en valor, proyectos de investigación, contemplan la problemática de conservación de los componentes estructurales del sitio, poco énfasis le ponen en la ejecución de los planes de manejo ambiental del proyecto, y le empiezan a prestar atención solo cuando el problema se vuelve muy evidente o irreversible.

Por problemas en la gestión de los residuos sólidos, en 2016 se puso en riesgo la denominación de Machu Picchu como Patrimonio Mundial, ya que el distrito de Machu Picchu no cuenta con un mecanismo de gestión apropiado. De darse un proyecto de desarrollo del turismo y puesta en uso social de Choquequirao, se debe prestar mucha atención desde el inicio y gestionar los residuos considerando las miles de toneladas anuales de basura que los potenciales visitantes al sitio dejarán como producto de su llegada y en algunos casos estadía.

Los beneficios que la sociedad percibe por efecto de cambios en la mejora de la conservación pueden verse multiplicados cuando son acompañados con inversiones en materia de medio ambiente y cultural. 
Para que el programa de inversiones a nivel de políticas públicas en conservación y turismo pueden implementarse, es importante que se instale una "autoridad autónoma" liderada por la DDC y el responsable del ACR, de tal manera se logre gestionar los recursos necesarios para la implementación de las políticas.

La "Autoridad Autónoma del ACR", tendría la facultad de manejar sus recursos económicos, la misma que debe ser creada con una norma de mucha jerarquía, a nivel de Ley, de tal manera que se sustente los beneficios sociales que genera el ACR en la misma zona. Actualmente las autoridades responsables del ACR, no cuentan con las capacidades financieras para implementar, uno de sus instrumentos de gestión, muy importante, "El Plan Maestro de Choquequirao", Los responsables del Área y la DDC, deben buscar fuentes de financiamiento para cada uno de sus programas de inversión, ya que estos proyectos muestran una rentabilidad social y económica aceptable. Actualmente el Perú tiene una cartera de fuentes de financiamiento como la Ley $\mathrm{N}^{\circ} 29230$ "Ley de obras por impuestos", entre las principales (Diario Oficial el Peruano, 2006).

\section{Literatura citada}

Buckley, R. 2004. Impacts positive and negative: links between ecotourism and environment. En: Buckley, R. (Ed.), Environmental Impacts of Ecotourism. CABI Publishing, New York, pp. 1-14.

Clark, J.; Clarke, R. 2011. Local sustainability initiatives in English National Parks: what role for adaptive governance? Land Use Policy 28:314-324.

Coppock, J. 1982. Tourism and conservation. Tourism Management 3:270-5.

Cummings, R.B. 1986. Valuing environmental goods: an assessment of the contingent valuation method. USA.

Diario Oficial el Peruano. 2010. D.S. $\mathrm{N}^{\circ}$ 022-2010-MINAM 2010, Establecen Área de Conservación Regional de Choquequirao, Diario Oficina el Peruano, Lima, Perú.

Diario Oficial el Peruano. 2004. Ley General del Patrimonio Cultural de la Nación - Ley 28296- , publicada en el Diario Oficial el 22 de julio de 2004, Lima - Perú.

Diario Oficial el Peruano. 2006. Reglamento de la Ley 29296 (DS 011-2006-ED), publicado el 2 de Junio de 2006.

Diario Oficial el Peruano. 2014. Reglamento de Intervenciones Arqueológicas (DS 003-2014-MC), publicado el 4 de Octubre de 2014.

Garcia, M.; De la Calle, M.; Minguez, M. 2011. Capacidad de carga turística y espacios patrimoniales. Aproximación a la estimación de la capacidad de carga del Conjunto Arqueológico de Carmona (Sevilla, España). Boletín de la AGE 57:219241.

Garayo, U. 2001. Los espacios naturales protegidos: entre la conservación y el desarrollo. En: Lurralde, España 24 (2001), p 271-293.

Glaser, M.; Baitoninsigh, W.; Ferse, S.; Neil, M.; Deswandi, R. 2010. Whose sustainability? Top-down participation and emergent rules in marine protected area management in Indonesia. Mar. Policy 34:1215-1225.

Halffter, R.G. 2011. Reservas de la Biosfera: Problemas y Oportunidades en México. Acta Zoológica Mexicana 27(1):177-189.

Hernandez, S. 1998. Valoración de los servicios ambientales de un programa de arborización "Programa Hojas 
Verdes", en el Parque Simón Bolivar. Santa Fé de Bogotá: Instituto de Investigación de Recursos Biológicos Alexander Von Humbolt.

Kousis, M. 1989 . Tourism and the family in a rural Cretan community. Annals of Tourism Research 16:318-333.

Kuvan, Y.; Akan, P. 2005. Residents' attitudes toward general and forestrelated impacts of tourism: the case of Belek, Antalya. Tourism Management 26:691-706.

Lorenzo, J. 1989. "México". En Cleere, H. (Ed.).

Lorenzo, J. 1989. "México". En Cleere, H. (Ed.). Approaches to the Archaeological Heritage. Cambridge University Press. Cambridge, NY, EUA.

Luttrel, C.; Schrecjenberg, K.; Peskett, L. 2007. The implications of carbon financing for pro-poor community forestry. In: Brown, D. (Ed.), Forestry Briefing, vol. 14. Overseas Development Institute, London, UK.

Ministerio de Cultura. 2016. Resolución 030-2016, Aprueban nuevos valores del boleto por concepto de visitas o ingreso a los Monumentos Arqueológicos Prehispánicos $\mathrm{y}$ Museos, integrantes del Patrimonio Cultural de la Nación para su aplicación en el Ejercicio Fiscal 2017.

MINCETUR. 2017. Plan Copesco para la formulación, coordinación, dirección, ejecución y supervisión deproyectos de inversión de interés turístico a nivel nacional, MINCETUR, Lima, Perú.

Morales, F. 2007. El aporte de la Áreas Naturales Protegidas a la Economía Nacional. Lima: Biblioteca Nacional, INRENA.

Moreno, M. 2004. Patrimonio cultural, puesta en valor y uso. Una reflexión. Disponible en URL:http://www.fulp. ulpgc.es/newweb/publicaciones/ vectorplus/articulos/vector20 04.pdf Leído el 21/10/2012.

Newsome, D.; Moore, S.; Dowling, R. 2002. Natural Area Tourism: Ecology, Impacts and Management. Channel View Publications, Sydney

Ortiz de Orue, H. 2017. Borrador Tesis Doctoral en Economía de los Recursos Naturales y Desarrollo Sustentable titulada Estimación de los beneficios sociales reportados por mejoras en conservación y en Turismo en el área natural de Choquequirao - Cusco, Perú, Universidad Nacional Agraria La Molina, Lima, Perú.

Pickering, C.; Hill, W. 2007. Impacts of recreation and tourism on plant biodiversity and vegetation in protected areas in Australia. Journal of Environmental Management 85:791-800.

Proyecto Especial PLAN COPESCO. 2017. Plan Operativo Institucional. Disponible en http://www.copesco. gob.pe/attach/docsgestion/11.1\%20 P la n \% 20 Operativo\% 20 Institucional\%202017.pdf

PROMPERU. 2013. Perfil del Turista Extranjero: Turismo en cifras. LimaPerú: Prom Perú.

Quadri, G. 2006. Políticas públicas: Sustentabilidad y Medio ambiente. Cámara de Diputados. Tecnológico de Monterrey y Porrúa, México, p 190-200.

Romano, G.; Serrano, R. 2013. Estrategias de control de impactos turístico en las áreas naturales protegidas y zonas arqueológicas del Quintana Roo, México, Revista Cultur. www.uesc. br. 
SINANPE. 1999. Estrategia Nacional para las Areas Naturales. Lima-Perú.: Plan Director

Trigger, B. 1989. A History of Archaeological Thought. Cambridge University Press. Cambridge, NY, EUA.

Williams, P.; Ponsford, I. 2009. Confronting tourism's environmental paradox: Transitioning for sustainable tourism. Futures, 41, 396-404

Wunder, S. 2005. Payment for Environmental Services: Some Nuts and Bolts. CIFOR Occasional Paper. Center for International Forestry Research, Indonesia. URL: www.cifor.cgiar.org/ publications/pdf_files/OccPapers/ OP-42.pdf. 